\title{
Attribute abstraction, feature- dimensionality, and the scaling of product similarities
}

\author{
Michael D. Johnson * \\ School of Business Administration, University of Michigan, Ann \\ Arbor, MI 48109, USA \\ Donald R. Lehmann * \\ Graduate School of Business, Columbia University, New York, \\ NY 10027, USA
}

\section{Claes Fornell * and Daniel R. Horne * \\ School of Business Administration, University of Michigan, Ann Arbor, MI 48109, USA}

Recejved September 1990

Final version received July 1991

This paper examines the attributes that consumers use when making product similarity judgments and their effect on similarity scaling. Previous research suggests that concrete brands are judged using dichotomous features while more abstract product categories are judged using continuous dimensions. This, in turn, suggests that the appropriateness of spatial scaling increases relative to tree scaling as one moves from brands to product categories. The results of two studies support an increase in the fit of spaces relative to trees from brands to categories. However, the abstractness of the judgments appears to be driving the effect, not the use of features or dimensions.

Correspondence to: Michael D. Johnson, Associate Professor of Marketing, School of Business Administration, University of Michigan, Ann Arbor, MI 48109, USA.

* The authors thank C. Whan Park, the Editor, and two anonymous reviewers for their constructive comments on earlier versions of the paper. The financial support of the University of Michigan's School of Business Administration is also gratefully acknowledged.

Intern. J. of Research in Marketing 9 (1992) 131-147

North-Holland

\section{Introduction}

Consumers cognitively represent, describe, or "think about" alternatives in terms of attributes. These attributes resemble either distinct features or more continuous dimensions (Garner, 1978; Restle, 1959; Tversky, 1977; Tversky and Gati, 1978). Attributes also vary from the concrete to the abstract (Howard, 1977; Johnson, 1984; Rosch, 1975). Consumers' judgments of interproduct similarity are likely to vary, therefore, in their reliance on features or dimensions as well as their concreteness-abstractness.

This paper examines the feature-dimensionality and the concreteness-abstractness of the attributes processed in a similarity judgment task. We further examine the impact that these factors have on the scaling of product similarity judgments. It has been suggested that dimension-based judgments are more consistent with spatial scaling techniques while feature-based judgments are more consistent with tree-scaling procedures (Pruzansky et al., 1982; Sattath and Tversky, 1977). Moreover, attribute representations may become more dimension-based and less feature-based at higher levels of abstraction (Johnson and Fornell, 1987). Thus the appropriateness of spatial scaling relative to tree scaling may increase as one moves from concrete brands to more abstract product categories.

Two studies are reported. The first uses self-reports to examine the attributes that consumers use to judge similarity at the brand and category levels and their effect on simi- 
larity scaling. The results support an increase in attribute abstraction from brands to categories and a related increase in the fit of spaces relative to trees. Yet the observed scaling differences are not due to differences in feature-based versus dimension-based processing as previously supposed. The second study, which does not rely on self-reports, confirms the main scaling results from study one. Together the studies suggest that spatial scaling captures consumers' product perceptions at an inherently more abstract level than does tree scaling.

\section{Features, dimensions, and abstraction}

Attributes have been traditionally described as resembling either features or dimensions (Garner, 1978; Myers and Shocker, 1981; Park, 1978; Restle, 1959; Tversky, 1977). Features are dichotomous attributes which objects either have or do not have (e.g., anti-lock brakes) while dimensions are attributes on which objects vary as a matter of degree (e.g., safety). However, even inherently continuous attributes may be recalled and processed as features (Garner, 1978; Prinz and Scheerer-Neuman, 1974; Tversky, 1977). For example, consumers may simply describe automobiles as safe or not safe even though they vary continuously on safety (for a similar view see Park (1978)). This underscores an important conceptual difference between the inherent feature-dimensionality and the processed feature-dimensionality of an attribute representation.

Recent research suggests that inherent feature-dimensionality is a direct function of the level of abstraction of the products and attributes involved (Johnson and Fornell, 1987). ${ }^{1}$ Following Howard (1977) and Rosch (Rosch, 1975; Rosch et al., 1976), more abstract, superordinate products are described using more abstract attributes. These more abstract attributes are themselves inherently continuous (Johnson and Fornell, 1987). By definition, abstract attributes more completely describe objects than do concrete attributes (Paivio, 1971). For example, an automobile's degree of "safety" captures and conveys information regarding a range of more concrete attributes, many of which are dichotomous features (e.g., air-bag, anti-lock brakes). Thus an abstract attribute, by its nature, has more levels or continuity than a feature. This does not imply that continuous attributes are necessarily abstract. Products are routinely described on a number of continuous concrete dimensions (e.g., size, weight, height). At the same time, it is difficult to think of a truly abstract attribute (e.g., safety, durability, necessity, fun) that is inherently dichotomous.

Johnson and Fornell (1987) report on two studies that support an increase in inherent feature-dimensionality with product abstraction. The studies used recall and association tasks respectively to explore the attributes that consumers use to describe increasingly abstract products, from brands, to categories, to superordinate categories. In both studies subjects associated more abstract attributes with more abstract products and these abstract attributes were more likely to resemble inherently continuous dimensions.

What remains unclear is just how these continuous dimensions are processed in the course of a similarity judgment task. The inherent dimensionality of more abstract attributes may be reflected directly in the processed feature-dimensionality of consumers'

Concreteness-abstractness is defined as the directness with which attributes describe particular objects or products and is equated with the specificity-generality of terms as well as the subordination-superordination of product categories (Paivio, 1971; Rosch, 1977). More abstract attributes, such as a product's safety or nutritional value, describe products more indirectly and completely. More concrete attributes, such as an automobile's braking system or a candy bar's ingredients, describe products more directly and specifically. 
judgments. However, differences in product experience between brands and categories may seriously mitigate this effect. Alba and Hutchinson define experience or familiarity as "the number of product-related experiences that have been accumulated by the consumer" while expertise reflects how this experience affects a consumer's "ability to perform product-related tasks" (Alba and Hutchinson, 1987, p. 411) Put differently, experience constitutes a consumer's actual interaction with particular products (Fornell et al., 1985).

Generally, consumers should have more experience with an array of product categories than with an array of brands in any one of those categories (Howard, 1977). It is not necessary to purchase and consume multiple instances or members of a category to have experience with the category. Each brand level experience is simultaneously an experience with the category. As a result, the more abstract or categorical the stimuli, the more experiences a consumer should be able to draw upon. Consumers should, for example, have greater experience with snack foods or beverages than with specific brands in a particular snack food or beverage category (e.g., specific candy bars or soft drinks).

Greater experience may, in turn, allow consumers to process inherently continuous dimensions as features at the product category level. A wide range of studies in psychology and consumer behavior demonstrate how product familiarity increases consumers' ability to isolate that information which is most important and relevant to the task at hand (see Alba and Hutchinson, 1987). As a result, experienced consumers may be better able to "chunk" or categorize inherently continuous dimensions into value ranges. As Park (1978) argues, the categorization of continuous product dimensions in a judgment or choice task is not straightforward and may only be learned through experience. These categorized value ranges allow con- sumers to efficiently process an inherently continuous dimension as a set of nested features (Tversky, 1977).

Thus the degree to which attributes are processed as features rather than dimensions in a similarity task may increase with experience. Park (1976) demonstrates a similar effect in a choice task. He found that subjects' product evaluation judgments became more consistent with conjunctive and disjunctive decision rules at higher levels of product familiarity. Such rules often require consumers to categorize continuous dimensions into acceptable and unacceptable value ranges or, in our framework, to process inherent dimensions as features.

Figure 1 summarizes the relations among the constructs of interest here. Stimulus abstraction refers to the categorical level of the products involved (e.g., brands versus categorics). The fcature-dimensionality construct represents processed feature-dimensionality, or the degree to which processed attributes resemble continuous dimensions as opposed to dichotomous features. Moving from brands to categories should result in more abstract attribute representations and allow consumers to rely on a greater store of experience. Recall that abstract attributes are inherently dimensional. To the degree that inherent feature-dimensionality carries over and increases processed feature-dimensionality, attribute abstraction should positively affect feature-dimensionality in Fig. 1. In contrast, experience should have a direct effect on processed feature-dimensionality which is negative rather than positive. Consumers' greater experience with categories should facilitate feature-based processing and hence reduce feature-dimensionality.

Not included in Fig. 1 is the possible effect of experience on attribute abstraction. This effect may be very different depending on whether product categories or brands are involved. As experience grows, consumers should store and be able to process more 


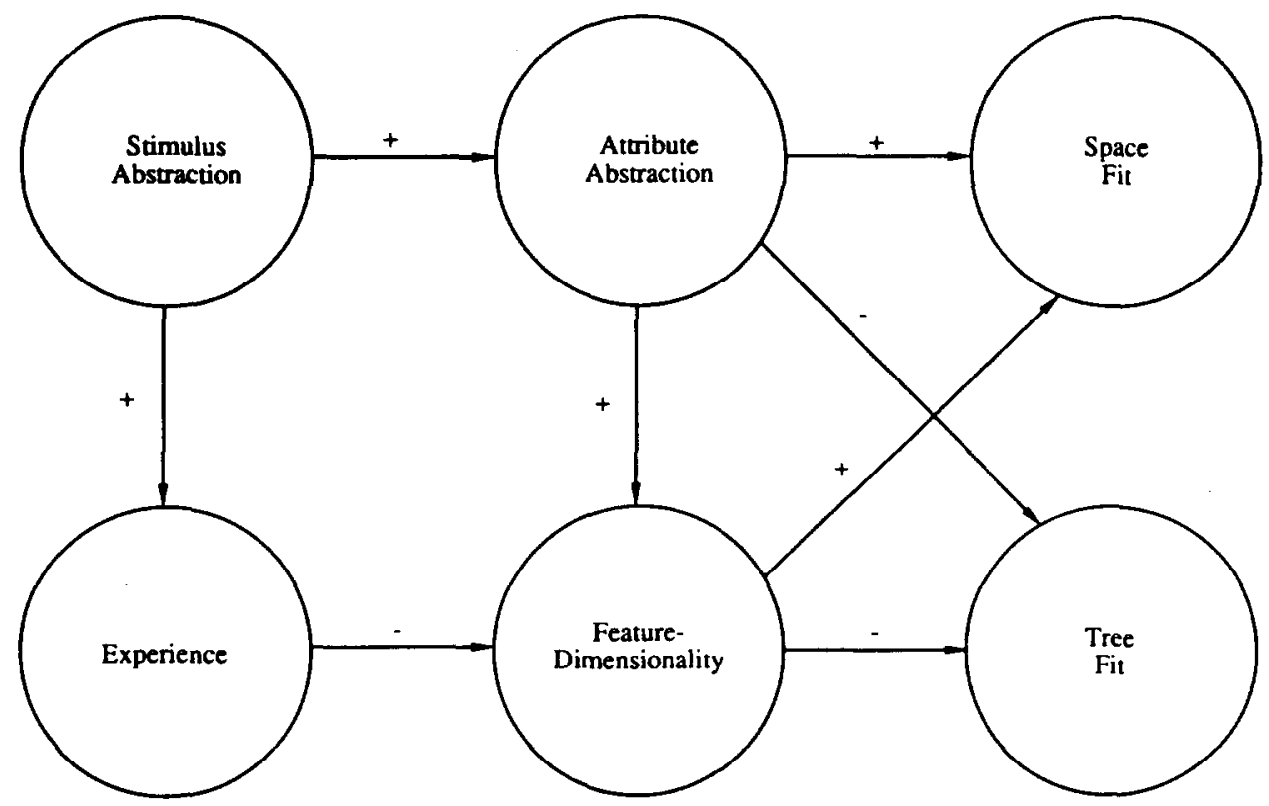

Fig. 1. Conceptual model.

concrete information regarding product categories (Bettman and Sujan, 1987; Biehal, 1983; Sujan, 1985; Walker et al., 1987). As consumers become more experienced with brands, they should store and be able to process more abstract, summary information (Bettman and Jacoby, 1976; Johnson and Russo, 1984; Wright, 1975) or "brand concepts" (Howard, 1977). Although no general effect is predicted across levels of stimulus abstraction, possible effects will be examined at the brand and category levels separately.

\section{Spaces and trees}

The abstractness and feature-dimensionality of consumers' similarity judgments may have important implications for similarity scaling. Researchers utilize two general classes of techniques to scale similarity judgments, spatial scaling and tree scaling or clustering. Spatial scaling, more specifically multidimensional scaling, represents products as varying on a small number of continuous dimensions in some underlying geomet- ric space (Kruskal, 1964; Shepard, 1962). Tree scaling and clustering schemes, including additive tree scaling (Sattath and Tversky, 1977), extended tree scaling (Corter and Tversky, 1986), hierarchical clustering (Johnson, 1967), and additive clustering (Shepard and Arabie, 1979), typically represent stimuli as external nodes in a tree structure. Perceptual distance in the tree is proportional to the length of the paths separating the stimuli. The following sections describe just how the feature-dimensionality and abstractness of consumers' judgments may affect the fit of these techniques.

\section{Feature-dimension isomorphism}

A number of researchers hypothesize an isomorphism or correspondence between tree and space scaling and attribute feature-dimensionality (Carroll, 1976; Johnson and Fornell, 1987; Pruzansky et al., 1982; Tversky and Hutchinson, 1986). Accordingly, techniques that produce spatial representations, such as multidimensional scaling, presume more continuous dimensions. Techniques 
that produce tree-like structures, such as additive or extended tree scaling, presume more feature-based representations. Put simply, geometric spaces are more continuous and dimensional while clustering schemes are more directly interpretable using product features. Thus spatial scaling should be more appropriate for dimension-based judgments while tree scaling should be more appropriate for feature-based judgments.

Johnson and Fornell used this isomorphism to predict scaling differences across levels of product abstraction. They argued that when moving from brands to categories, judgment processing becomes more abstract and dimension-based. This increase in processed feature-dimensionality should increase space fit relative to tree fit. To test the prediction, they examined the effects of abstraction on three different types of scaling solutions, two- and three-dimensional MDS and additive tree scaling via ADDTREE. Aggregate-level similarity judgments were examined for stimulus sets which ranged from concrete brands to more abstract product categories. Principal components analysis was used to operationalize level of abstraction; fewer, more explanatory latent roots were equated with more abstract representations. The results revealed an increase in the ability of the spaces to fit the similarity judgments relative to the additive trees as abstraction increased.

However, the observed scaling differences may have nothing to do with the processed feature-dimensionality of the judgments. As discussed earlier, it is not clear whether dimension-based processing increases from brands to categories as Johnson and Fornell assume. More importantly, belief in an isomorphism or direct correspondence between processed feature-dimensionality and treebased versus spatial scaling is far from universal. For example, Torgerson argues that a "spatial representation of similarity relations among stimuli neither requires nor even im- plies a dimensional interpretation" (Torgerson, 1986, p. 57). Hence it may not be appropriate to view either multidimensional scaling or tree scaling as presuming particularly dimensional or feature-based representations. There are at least two alternative explanations for the observed scaling differences which have not been explored: a latent attribute difference and an aggregation effect.

\section{Latent attribute difference}

The latent attribute explanation rests on a very basic difference between space and tree scaling. Spatial scaling, being conceptually similar to factor analysis, captures relatively abstract or latent attributes (Shepard, 1972). Like factor analysis and principal components, MDs is very much a data reduction technique. It describes differences among products on a small number of more abstract or latent constructs. In contrast, clustering and tree scaling simply reveal the salient attributes or aspects that underlie perceptions (Johnson, 1967; Sattath and Tversky, 1977). Each branch or cluster may be interpreted as a different concrete attribute of the products.

Thus an increase in space fit relative to tree fit from brands to categories may be a direct function of attribute abstraction (see Fig. 1). Notice that both the "isomorphism" argument and the alternative "latent attribute" argument predict a similar end-result. As consumers move from brands to categories and judge products using more abstract attributes, the fit of spaces should improve relative to the fit of trees. However, according to the latent attribute argument, any scaling differences are due directly to increases in attribute abstraction. According to the isomorphism argument, processed feature-dimensionality mediates any scaling differences (see Fig. 1). Both of these predictions are examined in Study One. 


\section{Aggregation effect}

A different explanation for Johnson and Fornell's scaling results is that they are an artifact of aggregation. Their study, as well as previous studies that support a scaling isomorphism (Pruzansky et al., 1982), looked only at aggregate level similarity judgments. From a scaling standpoint, there may be no true isomorphism or direct correspondence between processed feature-dimensionality and similarity scaling techniques. Instead, there may only be a more underlying similarity between inherent feature-dimensionality and tree-based versus spatial scaling, which is revealed as perceptions are aggregated across consumers.

Consider two groups of consumers, the first of which uses the same abstract dimensions to judge a set of product categories while the second uses the same concrete features to judge a set of brands. Assume that the attributes are equally weighted. In the first group, many of the abstract dimensions are categorized and processed as features. Yet it is unlikely that the consumers operationalize these features in exactly the same way. Even relatively homogeneous consumers should, for example, vary in their labeling of snack foods as healthy or not, or automobiles as safe or not. The inherently continuous nature of the underlying perceptual dimensions may only materialize as judgments are aggregated across subjects.

Aggregation should not have the same effect on the feature-based judgments of the second group. Inherently dichotomous or feature-based perceptions should remain feature-based upon aggregation. For example, either at an individual or at an aggregate level the diet versus non-diet nature of soft drinks, or whether an automobile is installed with anti-lock brakes is easily scaled or modeled using features. Whether individual or aggregate perceptions are involved, percep- tual judgments should continue to reflect features.

Thus, at an aggregate level, individual differences may make product category perceptions appear to be more continuous than brand perceptions. Space fit may increase relative to tree fit from brands to categories, but as an artifact of the aggregation of abstract features. This possibility is examined in Study Two.

\section{Study One}

Study One was conducted to test the relationships in Fig. 1. The data for the test were collected using a two-part questionnaire. In part one, subjects were asked to provide measures of their experience with a set of stimuli. In part two, subjects provided judgments of product similarity for these stimuli and were asked to report on the aspects or attributes that they used in making their judgments.

Stimulus abstraction and the similarity judgments

Five sets of stimuli were used to operationalize two different levels of stimulus abstraction or categorization $\left(C_{1}\right)$. Two sets, soft drinks and candy bars, are very concrete and represent brands from the same product categories. The three remaining stimulus sets, beverages, snacks, and lunch products, represent more abstract, product category alternatives. The specific stimuli within each of these five stimulus sets are presented in Table 1. Each stimulus set contained twelve alternatives. Each subject provided paired-comparison similarity ratings for one of the five stimulus sets listed in Table 1. Each possible pair of the twelve alternatives was rated on an eleven-point scale ranging from 0 (Very Dissimilar) to 10 (Very Similar) for a total of sixty-six judgments. 


\section{Experience measures}

Experience measures were collected from each subject for each product in the subject's stimulus set. The measures were chosen to be as comparable across stimulus sets as possible. Experience was measured using four objective indicators: average frequency of consumption $\left(E_{1}\right)$, average frequency of purchase $\left(E_{2}\right)$, average recency of consumption $\left(E_{3}\right)$, and average recency of purchase $\left(E_{4}\right)$. Each subject was asked to rate his or her frequency (recency) of consumption (purchase) for each of the twelve products on separate five point scales (past day $=1$, past week $=2$, past month $=3$, past year $=4$, and year or more $=5$ for the recency of purchase and recency of consumption questions; every day $=1$, every week $=2$, every month $=3$, every year $=4$, and never $=5$ for the frequency of purchase and frequency of consumption questions). Each of these four measures constitutes a qualitatively different aspect of a consumer's experience with particular products which, taken together, constitute the subject's experience.

Processed attributes: attribute abstraction and feature-dimensionality

Immediately after collecting the similarity judgments, subjects were asked to recall the product aspects or characteristics that they processed in the course of the judgment task. They were asked to recall and list as many aspects as they could "which they used to compare the alternatives". The subjects were told that they could refer to their judgments

Table 1

Stimulus sets

a. Brand level stimuli

\begin{tabular}{|c|c|c|}
\hline Soft drinks & Candy bars & \\
\hline $\begin{array}{l}\text { Sprite } \\
\text { Seven Up } \\
\text { Diet Sprite } \\
\text { Diet Seven Up } \\
\text { Orange Crush } \\
\text { Diet Orange Crush } \\
\text { Coke Classic } \\
\text { New Coke } \\
\text { Pepsi } \\
\text { Cherry Coke } \\
\text { Diet Coke } \\
\text { Diet Pepsi } \\
\end{array}$ & $\begin{array}{l}\text { Three Musketeers } \\
\text { Mars Bar } \\
\text { Milky Way } \\
\text { Snickers } \\
\text { M\& M Plain } \\
\text { M \& M Peanut } \\
\text { Hershey's Plain } \\
\text { Hershey's Almond } \\
\text { Nestle's Crunch } \\
\text { Reece's Peanut Butter Cups } \\
\text { Twix Caramel } \\
\text { Kit Kat }\end{array}$ & \\
\hline \multicolumn{3}{|c|}{ b. Category level stimuli } \\
\hline Beverages & Snacks & Lunch products \\
\hline $\begin{array}{l}\text { Ice Cream Soda } \\
\text { Milk Shake } \\
\text { Chocolate Milk } \\
\text { Milk } \\
\text { Fruit Juice } \\
\text { Lemonade } \\
\text { Soft Drink } \\
\text { Diet Soft Drink } \\
\text { Club Soda } \\
\text { Iced Tea } \\
\text { Bottled Water } \\
\text { Iced Coffee }\end{array}$ & $\begin{array}{l}\text { Popcorn } \\
\text { Nacho Chips } \\
\text { Crackers } \\
\text { Potato Chips } \\
\text { Cheese } \\
\text { Grapes } \\
\text { Apple } \\
\text { Yogurt } \\
\text { Ice Cream } \\
\text { Cookie } \\
\text { Candy Bar } \\
\text { Brownie }\end{array}$ & $\begin{array}{l}\text { Carrot } \\
\text { Apple } \\
\text { Fruit Juice } \\
\text { Yogurt } \\
\text { Milk } \\
\text { Ice Cream } \\
\text { Cookie } \\
\text { Candy Bar } \\
\text { Soft Drink } \\
\text { Pizza } \\
\text { Chicken Sandwich } \\
\text { Hamburger }\end{array}$ \\
\hline
\end{tabular}


as a guide to aid them in this task. Immediately after listing each aspect, subjects rated whether the aspect was something that the products "either had (were) or did not have (were not)" (i.e., a feature) or "varied on as a matter of degree" (i.e., a dimension).

Our choice of these retrospective self-reports for eliciting the content of the subjects' processing was based on two primary considerations. First, it was felt that concurrent methods for eliciting the content of processing, such as concurrent protocols, might be reactive in a similarity judgment context. Being retrospective, the self reports should not interfere with the similarity judgments themselves. Second, we had the subjects list their attributes immediately after the similarity task. Such immediate post-task reports should provide valid insight into the content of the subjects' judgment processing (Ericsson and Simon, 1980). Minimizing the delay should minimize any subjective interpretation or transformation of the information. A longer delay might, for example, increase the reporting of inherent as opposed to processed feature-dimensionality.

Two measures of processed feature-dimensionality were operationalized for each subject. The first, derived from the subjects' own ratings, was the proportion of elicited attributes which the subject rated as dimensions $\left(D_{1}\right)$. The second measure was obtained by having a separate judge (one of the authors) similarly classify each of the attributes as a feature or dimension and again measuring the fraction that were dimensions for each subject $\left(D_{2}\right)$. Both are straightforward positive measures of feature-dimensionality based on classification judgments (see Johnson and Fornell, 1987).

The attributes were then rated by separate judges to provide measures of attribute abstraction. The listed attributes were assembled and rated independently by four judges. Each attribute was rated on a ten-point scale ranging from 0 (Very Concrete) to 10 (Very
Abstract). Averaging such judgments has been shown to produce reliable and consistent measures of concreteness-abstractness (Johnson, 1984, 1988; Johnson and Fornell, 1987). The judges included two naive judges and two of the authors. Given the potential differences between the authors and the naive judges, the ratings of the two authors were combined into one average and the ratings of the two naive judges were combined into a second average. Each of these concreteness-abstractness measures were then used to calculate the average abstractness of the aspects considered by each subject to provide two separate measures of attribute abstraction $\left(A_{1}\right.$ and $\left.A_{2}\right)$.

\section{Space fit and tree fit}

Several measures of space and tree fit were operationalized. Two spatial solutions were obtained for each subject's judgments using MDs in both two and three dimensions (Roskam and Lingoes, 1970). Similarly, two tree scaling solutions were obtained for each subject, an ADDTREE and an EXTREE. ADDTREE is a heuristic additive tree procedure (Sattath and Tversky, 1977). EXTREE first constructs an additive tree and then searches for non-nested clusters that are overlaid on the tree (Corter and Tversky, 1986).

Several considerations prompted a focus on these particular solutions. Reliance on two- and three-dimensional MDS solutions is consistent with previous research (Johnson and Fornell, 1987; Pruzansky et al., 1982; Sattath and Tversky, 1977). They are also extremely common in applications (Cooper, 1983; Shepard, 1972). Additive trees use approximately the same number of independent parameters as a two-dimensional spatial configuration (Carroll, 1976; Sattath and Tversky, 1977; Pruzansky et al., 1982) while the increase in parameters from additive to extended trees is comparable to the extension from two- to three-dimensional spatial 
scaling (Corter and Tversky, 1986). Finally, including extended as well as additive trees does not limit the tree scaling solutions to nested attribute structures (see Corter and Tversky (1986) for a discussion).

Consistent with previous studies (Johnson and Fornell, 1987; Pruzansky et al., 1982; Sattath and Tversky, 1977) two measures of fit were obtained for each solution, Kruskal's monotonic stress and linear $R^{2}$. Stress is a measure of badness of fit while $R^{2}$ is a measure of goodness of fit. These measures were standardized, scaled in the same direction, and combined to arrive at a single measure of fit for each of the four individual-level solutions. This resulted in two measures of space fit, two-dimensional space fit $\left(S_{1}\right)$ and three-dimensional space fit $\left(S_{2}\right)$ and two measures of tree fit, ADDTREE fit $\left(T_{1}\right)$ and EXTREE fit $\left(T_{2}\right)$.

\section{Procedure}

As noted, the experience measures were collected in part one. The stimuli in each stimulus set were rated in one random order by half the subjects and in the reverse order by the remaining subjects. In part two of the questionnaire, the subjects first rated the similarity of each possible pair of products in the set. The sixty-six pairs were listed in one random order for half the subjects and the reverse order for the remaining subjects. The subjects were then asked to list separately the attributes or aspects they considered and classify each attribute as a feature or dimension.

An experimenter paced the subjects through each phase. The instructions were read aloud and the subjects were instructed to stop and wait for further instructions before beginning the next task. After filling out the first part of the questionnaire, the subjects took a break (fifteen minutes) and were then administered part two. The subjects were recruited from evening business classes and were run in groups of approximately 30 $(n=123)$. The subjects were paid for their participation. A small number of subjects did not provide usable responses (i.e., incomplete responses or degenerate MDS solutions) and were eliminated prior to analysis. This left $20,24,21,24$, and 27 subjects respectively for the soft drinks, candy bars, beverages, snacks, and lunch products $(n=116)$.

\section{Analysis}

Stimulus abstraction, attribute abstraction, feature-dimensionality, and space and tree fit were all modeled reflectively by their indicators. Recall that the four experience measures combine to form a subject's experience. They are, therefore, modeled as formative indicators of experience (Fornell, 1989). Given the size of the sample, the categorical nature of the stimulus abstraction construct, and the use of formative experience measures, partial least squares was used to estimate the model (Fornell, 1989; Fornell and Bookstein, 1982; Wold, 1982). Negative construct indicators (e.g., the experience scales) were directionally re-scaled so that, a priori, all indicators should be positively related to their constructs.

The structural model was initially estimated using all of the brand and category subjects $(n=116)$. Separate models were then estimated for those subjects judging brands (soft drinks and candy bars, $n=44$ ) and categories (beverages, snacks, and lunch products, $n=72$ ). These latter models included the potential direct effect of experience on attribute abstraction. Importantly, the models test the generality of the proposed relationships at each separate level of stimulus abstraction.

\section{Overall results}

The structural model estimation, including the path coefficients and indicator loadings, 
is presented in Fig. 2. Notice that the measurement loadings are all large and positive. Over 91 percent of the measurement model covariance is accounted for. The measurement loadings are all greater than the path coefficients, which supports the discriminant validity of the model.

The path coefficients involving stimulus abstraction, experience, attribute abstraction, and feature-dimensionality were as predicted. Stimulus abstraction had a positive effect on attribute abstraction (0.448). Attribute abstraction, in turn, had a positive effect on the feature-dimensionality of processed attributes $(0.276)$. Stimulus abstraction also had a positive effect on experience (0.794) which, in turn, had a negative effect on feature-dimensionality $(-0.320)$. Consistent with the model in Fig. 1, judging categories as opposed to brands allows con- sumers to draw on a greater base of product experience and results in the use of more abstract attributes. The subsequent featuredimensionality of these attributes increased with attribute abstraction yet decreased with experience. These results suggest that there is little or no difference in processed feature-dimensionality between brands and categories. To illustrate, we standardized and averaged the measurement indicators for the feature-dimensionality construct. The means were almost identical and even decreased slightly from brands $(0.09)$ to categories $(-0.06)$.

Moving to the scaling results, attribute abstraction had a positive effect on space fit relative to tree fit $(0.194$ versus -0.080$)$. This is consistent with the notion that spatial scaling captures inherently more abstract or latent attributes. Interestingly, the feature-

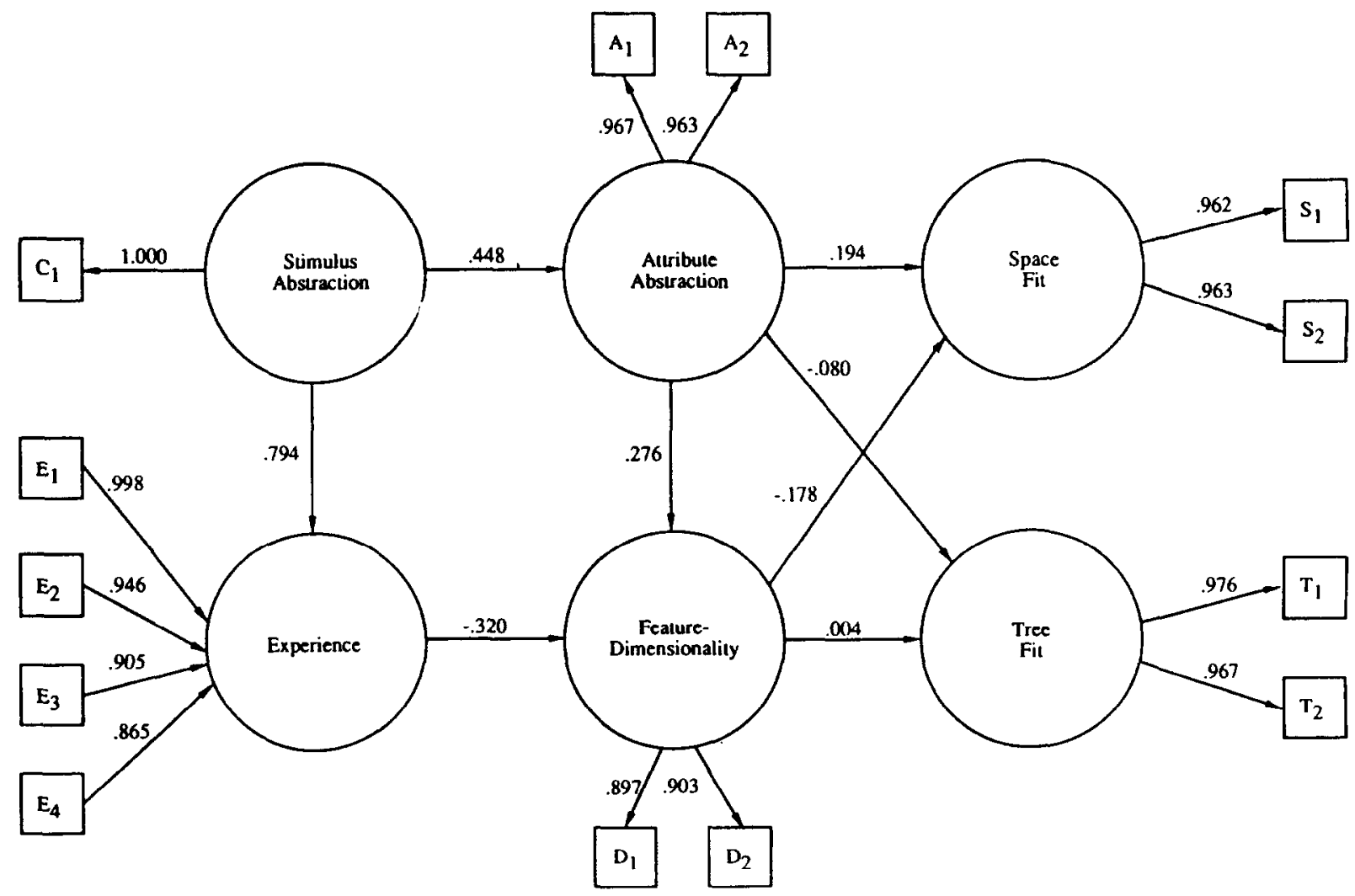

Fig. 2. Study One PLs model. 
dimensionality of processed attributes decreased space fit relative to tree fit $(-0.178$ versus 0.004), which is inconsistent with a strict isomorphism argument.

In addition to the PLS analysis, the relationships in Fig. 1 were examined using simple linear models. Multiple measures were standardized and averaged for each construct to provide single measures for the analyses. Consistent with the PLs output, the results show significant increases in experience $(F=156.99, p<0.001)$ and attribute abstraction $(F=28.433, p<0.001)$ from brands to categories. Feature-dimensionality showed a marginally significant increase with attribute abstraction $(F=3.011, \quad p<0.10)$ and a significant decrease with experience $(F=5.023, p<0.05)$. Finally, separate models examined the effects of attribute abstraction and feature-dimensionality on relative fit (i.e., space fit - tree fit). The fit of spaces increased relative to the fit of trees with attribute abstraction ( $F=8.042, p<0.005$ ). Relative fit did not vary significantly with feature-dimensonality.

\section{Brands versus categories}

Structural models were also estimated separately at the brand and category levels. A possible effect of experience on attribute abstraction was incorporated into each model. (Recall that experience may have a positive effect on attribute abstraction for the brands and a negative effect for the categories.)

The structural model results are very consistent with those in Fig. 2. In each case, the measurement loadings were large and positive and exceeded the path coefficients. For the brands, experience had a negative effect on feature-dimensionality $(-0.351)$ and a small but predicted positive effect on attribute abstraction (0.142). Attribute abstraction, in turn, had a positive effect on feature-dimensionality (0.304) and increased space fit (0.171) more than tree fit (0.047). Feature-dimensionality decreased space fit $(-0.245)$ more than tree fit $(-0.089)$. For the product categories, experience again had a negative effect on feature-dimensionality $(-0.334)$ and a small predicted negative effect on attribute abstraction $(-0.116)$. Attribute abstraction had a positive effect on feature-dimensionality $(0.165)$ and again increased space fit $(0.227)$ more than tree fit (0.087). Finally, feature-dimensionality again decreased space fit $(-0.131)$ more than tree fit $(0.070)$.

These results show how consistent the relationships in Fig. 2 are at each level of stimulus abstraction. Importantly, they show that the overall results are not a function of the particular operationalization of stimulus abstraction used in the study.

\section{Discussion}

The results paint an interesting picture of product similarity judgments and their scaling. Product categories are judged on the basis of more abstract attributes than are brands. Although these abstract attributes may be inherently continuous, there was no difference in processed feature-dimensionality at the brand and category levels. It appears that our subjects' experience with product categories allowed them to process product category attributes as features. This is consistent with Park's (1976, 1978) claim that experienced consumers are better able to dichotomize or categorize inherently continuous attributes for processing purposes. It is also consistent with the more general notion that experience leads to more complete processing heuristics (Alba and Hutchinson, 1987; Bettman and Park, 1980). Finally, the results fail to support a direct correspondence between processed feature-dimensionality and tree-based versus spatial scaling techniques. Instead, the results support a latent attribute difference between spaces 
and trees. Spatial representations appear to capture judgments at a more latent or abstract level than do tree scaling procedures.

\section{Study Two}

Study One provides a direct test of the latent scaling and isomorphism arguments. Yet the study has two particular limitations. It rests heavily on retrospective reports that may be limited in their ability to tap the content of processing (Nisbett and Wilson, 1977). By focusing on individual level protocols and judgments, Study One also fails to examine the possible effects of aggregation on space versus tree fit described earlier.

A second study was conducted with these limitations in mind. Existing proximity judgments were gathered from 20 different stimulus sets whose descriptive attributes were likely to vary in abstraction. These stimulus sets included the 5 sets used in study one and an additional 15 others that were available from existing research studies and projects. Separate judges were then asked to provide measures of stimulus abstraction for each stimulus set.

Consistent with previous research (Howard, 1977; Johnson, 1984; Paivio, 1971; Rosch, 1975; Rosch et al., 1976) and study one, we presume that the more abstract stimulus sets are judged on the basis of more abstract attributes. This allows an indirect observation of the effects of attribute abstraction on space and tree fit. Naturally the method is more removed from the content of the subjects' judgments than is Study One. We can not readily examine the effects of feature-dimensionality. However, study two does not rely on self-reports and the abstraction measures used here are more direct than the principal components measures used by Johnson and Fornell (1987).

Individual level judgments were available for each of the 20 stimulus sets involving a
Table 2

Stimulus sets for Study Two

\begin{tabular}{|c|c|c|c|}
\hline Stimulus set & Subjects & Stimuli & Abstraction \\
\hline Lunch products ${ }^{\text {a }}$ & 27 & 12 & 1.410 \\
\hline Dinner alternatives & 41 & 10 & 1.309 \\
\hline Children's toys & 23 & 13 & 1.244 \\
\hline Beverages & 31 & 10 & 1.066 \\
\hline Chevrolet cars and trucks & 9 & 12 & 1.003 \\
\hline Magazines & 31 & 11 & 0.812 \\
\hline Denim jeans & 24 & 12 & 0.289 \\
\hline Nonalcoholic beverages ${ }^{a}$ & 21 & 12 & 0.288 \\
\hline Snacks ${ }^{a}$ & 24 & 12 & 0.262 \\
\hline Sedans & 37 & 10 & 0.110 \\
\hline Female clothing stores & 14 & 10 & -0.145 \\
\hline Mid-sized cars & 11 & 11 & -0.145 \\
\hline Male clothing stores & 8 & 10 & -0.349 \\
\hline Soft drinks ${ }^{a}$ & 20 & 12 & -0.656 \\
\hline Restaurants & 35 & 12 & -0.732 \\
\hline Rental car agencies & 34 & 11 & -1.039 \\
\hline Fast food lunch restaurants & 29 & 10 & -1.127 \\
\hline Fast food dinner restaurants & 25 & 10 & -1.127 \\
\hline Cola soft drinks & 28 & 12 & -1.166 \\
\hline Candy bars ${ }^{a}$ & 24 & 12 & -1.306 \\
\hline
\end{tabular}

a Stimulus sets used in Study One.

total of 495 subjects. There were 25 subjects on average per set, varying from a low of 8 to a high of 41 , and the number of stimuli in the sets ranged from 10 to 13 . The stimulus sets are listed in Table 2. The judgments from each stimulus set were scaled at both the individual and aggregate levels. As in Study One, two spatial solutions were obtained using MDS in both two and three dimensions to model space fit while tree scaling solutions were obtained using ADDTREE and EXTREE to model tree fit. The judgments were first scaled at the individual level and the resulting fit measures aggregated for each stimulus set. The raw similarity judgments were also aggregated and then scaled to obtain aggregate level measures of fit. Following Study One, the space fit measures $\left(S_{1}\right.$ and $\left.S_{2}\right)$ and tree fit measures $\left(T_{1}\right.$ and $\left.T_{2}\right)$ are again composites of the monotonic stress and linear $R^{2}$.

This provided comparable individual and aggregate level observations for each of the 20 stimulus sets. Individual differences in 
perception do not affect our individual level fit measures as the fits are aggregated after scaling. They may, however, confound the aggregate-level fits. This prompted two separate structural model estimations, one for the individual judgments and one for the aggregate judgments. A measure of perceptual agreement $\left(G_{1}\right.$, being the average correlation of the raw similarity judgments across subjects) was included as a covariate for the aggregate level judgments to control for heterogeneity.

\section{Abstraction measures}

Two different measures of stimulus abstraction were operationalized. The first measure captures the categorical level of each stimulus set. Four judges familiar with the subordinate, basic, and superordinate category distinctions as described by Rosch et al. (1976) classified each stimulus set into one of these three levels. ${ }^{2}$ The resulting classifications were fairly well correlated for a threelevel categorical scale (average correlation of 0.47 , ranging from 0.25 to 0.70 ). Averaging across the four judges provides an initial measure of abstraction $\left(A_{1}\right)$.

A second measure of abstraction is based on the substitution-in-use of the stimulus alternatives (Shocker and Srinivasan, 1979; Srivastava et al., 1981). Similar products that are very substitutable in their use are likely to come from the same subordinate category and be described using the same concrete attributes. More dissimilar and less substitutable products may only be categorized at a very superordinate level and compared or described using more abstract attributes (Johnson, 1984). A convenience sample of

\footnotetext{
T'wo stimulus sets involved the exact same fast food restaurants rated under slightly different contexts (lunch versus dinner). Therefore, the category classification and substitution in use measures described here were collected for this one set of alternatives and applied to both data sets.
}

twenty judges rated the substitution-in-use of each of the twenty stimulus sets on a sevenpoint scale ranging from 1 (very substitutable) to 7 (not at all substitutable). Half of the judges rated the stimulus sets in one random order and the other half rated the sets in reverse order. One judge's ratings were eliminated due to consistent low or negative correlations with the other judges. For the remaining nineteen judges, 96 percent of the interjudge correlations were positive and the average correlation was 0.35. Although the individual level ratings were only moderately correlated, averaging across the judges' ratings should provide a reliable second measure of abstraction $\left(A_{2}\right)$. The resulting abstraction measures $A_{1}$ and $A_{2}$ were correlated 0.61 .

We standardized and averaged $A_{1}$ and $A_{2}$ to examine the face validity of abstraction differences across stimulus sets (see Table 2). Notice that the two beverage sets are more abstract than the soft drinks which, in turn, are more abstract than the cola soft drinks. The more abstract of the two beverage sets contains alcoholic as well as nonalcoholic beverages while the more concrete set contains only nonalcoholic beverages. A set containing cars as well as trucks is more abstract on the scale than two sets containing sedans and mid-sized cars respectively. Lunch products, which includes a range of both food and beverage stimuli, is the most abstract stimulus set while candy bars is the most concrete.

\section{Analyses and results}

Two structural models were estimated. The first examines the effects of abstraction on space and tree fit for the individual level judgments. This model provides a confirmatory test of the latent attribute differences observed in Study One. The second model examines the effects of abstraction on space and tree fit for the aggregate level judgments. Recall that this model includes a 


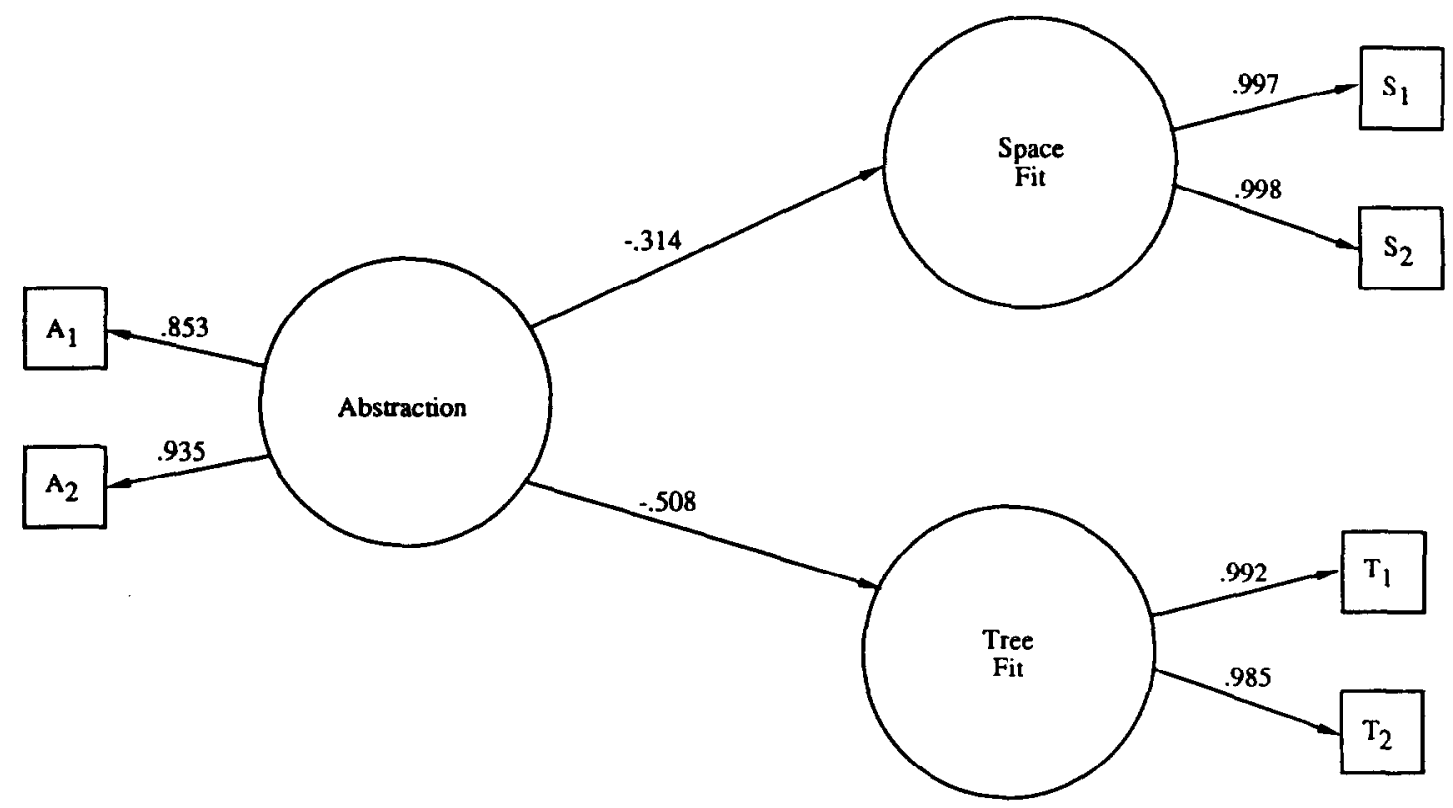

Fig. 3. Study Two: Individual level judgments.

measure of perceptual agreement to control for heterogeneity in the aggregated judgments. If the scaling differences observed by
Johnson and Fornell are simply the result of differences in abstraction, the two models should show similar results. If an aggregation

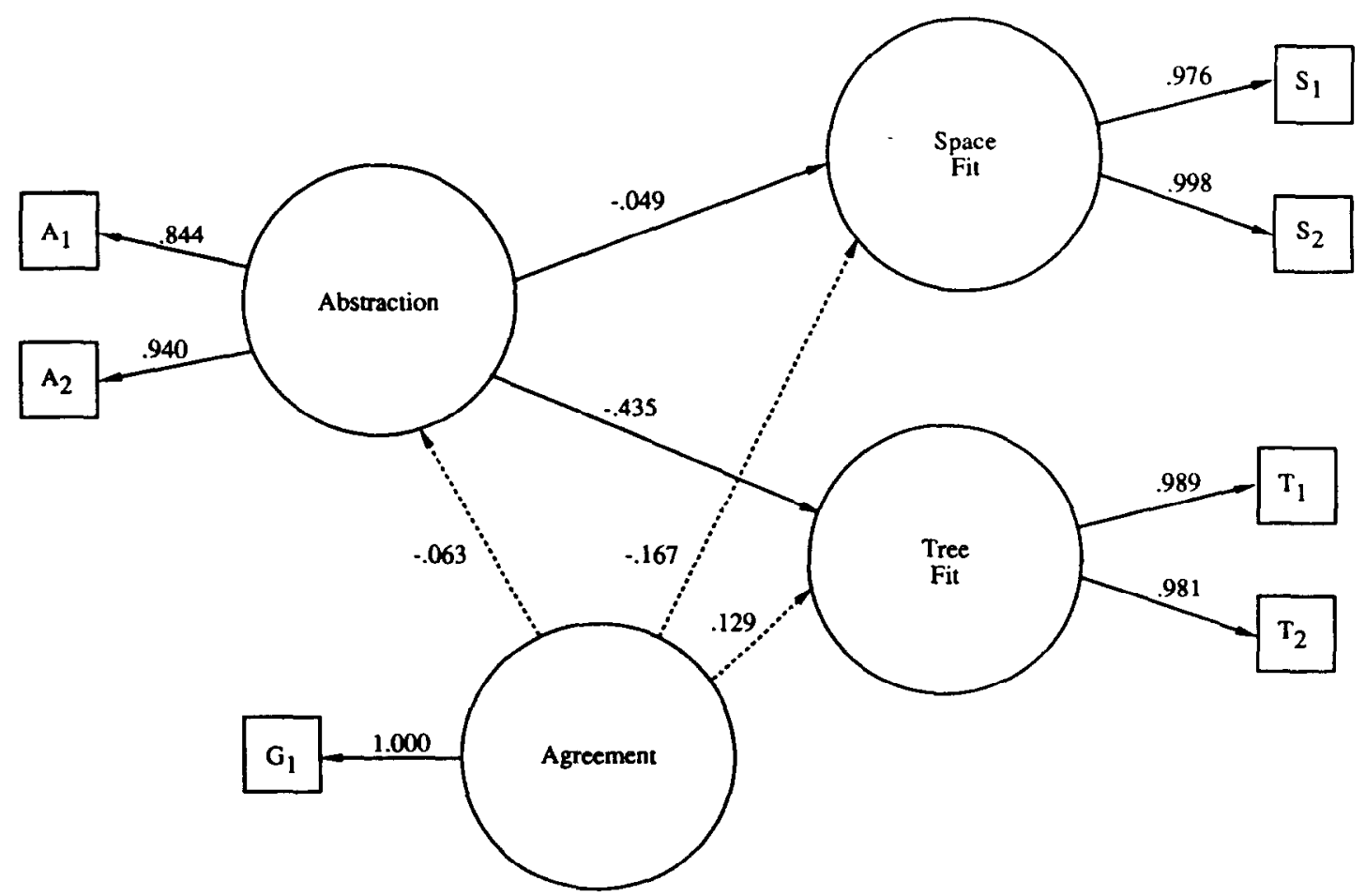

Fig. 4. Study Two: Aggregate level judgments. 
effect exists, then the relative advantage of spaces over trees with abstraction should be greater in the second model than in the first. Given the relatively small number of observations for analysis $(n=20)$, partial least squares was again used to estimate the structural models.

The results are shown in Figs. 3 and 4. Similar to Study One, the measurement indicators are large and positive for each model. The measurement model explains 92 percent of the measurement variance for both the individual and aggregate judgment models. The loadings again dominate the path coefficients supporting the discriminant validity of the models.

Consistent with Study One, the results support a latent attribute difference for the individual level judgments. Although abstraction reduced the fit of both the spaces and trees, it reduced the fit of the spaces less than the fit of the trees $(-0.314$ versus -0.508 ). One straightforward explanation for the overall decrease in fits is that judgment complexity may have increased with the superordination of the stimuli. For example, alcoholic and nonalcoholic beverages differ on a number of important abstract attributes (healthiness, taste, naturalness, potency) while soft drinks may differ on few important concrete attributes (cola versus noncola, diet versus nondiet). The critical result is that space scaling improves relative to tree scaling as abstraction increases.

The aggregate level results suggest that aggregation does affect the scaling techniques differently. The advantage of spaces over trees for the aggregate judgments $(-0.049$ versus -0.435$)$ is larger than the difference observed for the individual judgments which supports an aggregation effect.

To examine the results further, the individual and aggregate observations were pooled and single measures of abstraction and relative fit were derived. As in Study One, abstraction is a composite of $A_{1}$ and
$A_{2}$ while relative fit is the difference between the composite space fit and tree fit measures (space fit - tree fit). An analysis of variance model examined the effects of abstraction, level of aggregation, and an abstraction by level of aggregation interaction term on relative fit. Consistent with the latent attribute argument, the model revealed a significant main effect for abstraction on relative fit $(F=7.748, \quad p<0.01)$. A marginally significant abstraction by level of aggregation interaction $(F=2.692, p=0.11)$ provides some support for an aggregation effect. There was also a significant main effect increase in relative fit with aggregation $(F=5.751, p<0.05)$. This suggests that the aggregation effect may be more general than we thought.

Study Two confirms the latent scaling differences observed in Study One. The fit of spaces improves relative to the fit of trees when consumers judge products at a more abstract, categorical level. This finding is robust and not simply a result of the process measures used in Study One. Study Two also shows how the size of this abstraction scaling effect increases with aggregation. Although this second finding is more tentative, it suggests that previously reported support for an isomorphism between feature-dimensionality and similarity scaling (Johnson and Fornell, 1987; Pruzansky et al., 1982) may be attributed, in part, to aggregation.

\section{Summary and discussion}

Understanding when and why consumers use different types of attributes to judge product similarity is an important aspect of consumer and marketing research. The two studies presented here illustrate just how the content of consumers' similarity judgments changes from brands to product categories and how these changes affect similarity scaling techniques. 
Taken together, Studies One and Two support several conclusions. Judging categories as opposed to brands resulted in more abstract attribute processing. However, in contrast to previous predictions (Johnson and Fornell, 1987), there was no difference in the degree to which attributes were processed as features or dimensions at the brand and category levels. Consumers' relative experience with product categories allowed for featurebased processing of inherently abstract dimensions. As is the case for other judgment and choice tasks (Alba and Hutchinson, 1987; Park, 1978), experience results in more efficient similarity judgments.

The studies also support an abstraction scaling effect. Accordingly, the fit of spatial scaling improves relative to tree scaling with increases in stimulus and attribute abstraction. An important contribution of the present research is that the consumers' processing of features or dimensions does not drive this scaling effect as previously supposed. Instead, the data suggests that spatial scaling captures consumer perceptions at an inherently more abstract or latent level. The practical significance is that users may find spatial scaling techniques more useful for studying competition at the product category level. Tree scaling and clustering techniques may be more useful for studying competition among brands.

Finally the results of Study Two suggest that previous support for a feature-dimension scaling isomorphism may be, in part, an artifact of aggregation. Consumers are unlikely to operationalize features in an identical fashion. Upon aggregation, consumer perceptions may appear to be more continuous than they actually are. One implication is that constructs such as feature-dimensionality may only be discernable at the individual level.

Future research should explore other task or individual differences that might affect similarity judgments and scaling. Consumers' internal knowledge structures, their level of motivation, and their processing capacity may, for example, impact similarity judgments. A closer examination of the effects of aggregation on similarity judgments and their scaling also appears promising. Naturally the empirical studies reported here have limitations. It will be important to replicate our findings using qualitatively different stimuli, subjects, and methods.

\section{References}

Alba, J.W. and W.J. Hutchinson, 1987, Dimensions of consumer expertise. Journal of Consumer Research 13, 411454.

Bettman, J.R. and J. Jacoby, 1976. Patterns of processing in consumer information acquisition. In: B. Anderson (ed.), Advances in consumer research, Vol. 3, 315-320. Ann Arbor, MI: Association for Consumer Research.

Bettman, J.R. and C.W. Park, 1980. Effects of prior knowledge and experience and phase of the choice process on consumer decision processes: A protocol analysis. Journal of Consumer Research 7, 234-248.

Bettman, J.R. and M. Sujan, 1987. Effects of framing on evaluation of comparable and non-comparable alternatives by expert and novice consumers. Journal of Consumer Research 14, 141-154.

Biehal, G.J., 1983. Consumers' prior experiences and perceptions in auto repair choice. Journal of Marketing 47, 82-91.

Carroll, J.D., 1976. Spatial, non-spatial, and hybrid models for scaling. Psychometrika 41, 439-463.

Cooper, L.G., 1983. A review of multidimensional scaling in marketing research. Applied Psychological Measurement 7, 427-450.

Corter, J.E. and A. Tversky, 1986. Extended similarity trees. Psychometrika 51, 429-451.

Ericsson, K.A. and H.A. Simon, 1980. Verbal reports as data. Psychological Review 87, 215-251.

Fornell, C., 1989. The blending of theuretical and empirical knowledge in structural equations with unobservables. In: H. Wold (ed.), Theoretical empiricism. New York: Paragon House, 153-173.

Fornell, C., W.T. Robinson, and B. Wernerfelt, 1985. Consumption experience and sales promotion expenditure. Management Science 31, 1084-1105.

Fornell, C. and F.L. Bookstein, 1982. Two structural equation models: LISREL and PLS applied to consumer exit-voice theory. Journal of Marketing Research 19, 440-452.

Garner, W.R., 1978. Aspects of a stimulus: Features, dimensions, and configurations. In: E. Rosch and B. Lloyd (eds.), Cognition and categorization, 99-133. New York: John Wiley and Sons. 
Howard, J., 1977. Consumer Behavior: Application of theory. New York: McGraw-Hill.

Johnson, E.J. and J.E. Russo, 1984. Product familiarity and learning new information. Journal of Consumer Research $11,542-550$.

Johnson, M.D., 1984. Consumer choice strategies for comparing noncomparable alternatives. Journal of Consumer $\mathbf{R e}$ search $11,741-753$.

Johnson, M.D., 1988. Comparability and hierarchical processing in multialternative choice. Journal of Consumer Research 15, 303-314.

Johnson, M.D. and C. Fornell, 1987. The nature and methodological implications of the cognitive representation of products. Journal of Consumer Research 14, 214-228.

Johnson, S.C., 1967. Hierarchical clustering schemes. Psychometrika 32. 241254.

Kruskal, J.B., 1964. Multidimensional scaling by optimizing goodness of fit to a nonmetric hypothesis. Psychometrika 29. $1-27$.

Myers, J.H. and A.D. Shocker, 1981. The nature of productrelated attributes. Research in Marketing 5, 211-236.

Nisbett, R.E. and T.D. Wilson, 1977. Telling more than we can know: Verbal reports on mental processes. Psychological Review 84, 231-259.

Pajvio, A., 1971. Imagery and verbal processes. New York: Holt, Rinehart and Winston.

Park, C.W., 1976. The effect of individual and situation-related factors on consumer selection of judgmental models. Journal of Marketing Research 13, 144-151.

Park, C.W.. 1978. A conflict resolution choice model. Journal of Consumer Research 5, 124-137.

Prinz, W. and G. Scheerer-Neuman, 1974. Component processes in multiattribute stimulus classification. Psychological Research 37, 25-50.

Pruzansky, S., A. Tversky and J.D. Carroll, 1982. Spatial versus tree representations of proximity data. Psychometrika $47,3-24$

Restle. F.A., 1959. A metric and an ordering on sets. Psychometrika 24, 207-220.

Rosch. E., 1975. Cognitive representation of semantic categories. Journal of Experimental Psychology: General 104, 192-233.

Rosch, E., 1977. Human categorization. In: N. Warren (ed.) Siudies in cross-cultural psychology. Vol. I, 1-49. London: Academic Press.

Rosch, E., C.B. Mervis, W.D. Gray, D.M. Johnson and P. Boyes-Braem, 1976. Basic objects in natural categories. Cognitive Psychology 8, 382-439.
Roskam, E. and J.C. Lingoes, 1970. Minissa-1: A Fortran IV (G) program for the smallest space analysis of square symmetric matrices. Behavioral Science 15, 204-205.

Sattath, S. and A. Tversky, 1977. Additive similarity trees. Psychometrika 42, 319-345.

Shepard, R.N., 1962. The analysis of proximities: Multidimensional scaling with an unknown distance function, I and II Psychometrika 27, 125-140 and 219-246.

Shepard. R.N., 1972. Introduction to volume I. In: R. Shepard, A. Romney and S. Nerlove (eds.), Multidimensional scaling: Theory and applications in the behavioral sciences, Vol. I: Theory, 1-20. New York: Seminar Press.

Shepard, R.N. and P. Arabie, 1979. Additive clustering: Representation of similarities as combinations of discrete overlapping properties. Psychological Review 86, 87-123.

Shocker, A.D. and V. Srinivasan, 1979. Multi-attribute applications for product concept evaluation and generation: $A$ critical review. Journal of Marketing Research 15, 159-180.

Srivastava, R.K., R.P. Leone and A.D. Shocker, 1981. Market structure analysis: Hierarchical clustering of products based on substitution-in-use. Journal of Marketing 45, $38-48$.

Sujan. M.. 1985. Consumer knowledge: Effects on evaluation strategies mediating consumer judgment. Journal of Consumer Research 12, 31-46.

Torgerson, W.S., 1986. Scaling and Psychometrika: Spatial and alternative representations of similarity data. Psychometrika 51. 57-63.

Tversky, A.. 1977. Features of similarity. Psychological Review $84,327-352$.

Tversky, A. and I. Gati, 1978. Studies of similarity. In: E. Rosch and B. Lloyd (eds.), Cognition and categorization, Chapter 4, 79-98. New York: John Wiley and Sons.

Tversky, A. and J.W. Hutchinson, 1986. Nearest neighbor analysis of psychological spaces. Psychological Review 93, $3-22$.

Walker, B., R. Celsi, and J. Olson, 1987. Exploring the structural characteristics of consumers knowledge. In: M. Wallendorf and P. Anderson (eds.), Advances in consumer research. Vol. 14, 17-21. Provo, UT: Association for Consumer Rescarch.

Wold. H., 1982. Systems under indirect observation using PLS. In: C. Fornell (ed.), A second generation of multivariate analysis: Methods, 325-347. New York: Praeger.

Wright, P.. 1975. Consumer choice strategies: Simplifying vs. optimizing. Journal of Marketing Research 12, 60-67. 